\title{
Erratum
}

\section{Palladium-Catalyzed Silane/Siloxane Reductions in the One-Pot Conversion of Nitro Compounds into Their Amines, Hydroxylamines, Amides, Sulfonamides, and Carbamates}

Ronald J. Rahaim Jr., Robert E. Maleczka Jr.* Synthesis 2006, 3316.

The advanced online publication (e-First) version of this article included two incorrect structures, where the intended nitroarene starting materials were represented as anilines (graphic abstract and Table 7). These errors have been corrected for both the print and current online versions. 